\title{
Generation of Insulin-Producing Cells from Canine Adipose Tissue-Derived Mesenchymal Stem Cells
}

\author{
Takahiro Teshima $\mathbb{D D}^{1,2}$ Keiji Okamoto ${ }^{10},{ }^{1}$ Kazuho Dairaku $\mathbb{D}^{1},{ }^{1}$ Tomokazu Nagashima $\mathbb{D}^{\mathrm{D}},{ }^{3}$ \\ Masaki Michishita $\mathbb{D}^{2,3}$ Ryohei Suzuki ${ }^{1},{ }^{1}$ Hirotaka Matsumoto $\mathbb{D}^{1},{ }^{1}$ \\ and Hidekazu Koyama
}

${ }^{1}$ Laboratory of Veterinary Internal Medicine, Department of Veterinary Clinical Medicine, School of Veterinary Medicine, Faculty of Veterinary Science, Nippon Veterinary and Life Science University, 1-7-1 Kyonan-cho, Musashino, Tokyo 180-8602, Japan

${ }^{2}$ Research Center for Animal Life Science, Nippon Veterinary and Life Science University, 1-7-1 Kyonan-cho, Musashino, Tokyo 180-8602, Japan

${ }^{3}$ Laboratory of Veterinary Pathology, Department of Veterinary Pathobiology, School of Veterinary Medicine, Faculty of Veterinary Science, Nippon Veterinary and Life Science University, 1-7-1 Kyonan-cho, Musashino, Tokyo 180-8602, Japan

Correspondence should be addressed to Takahiro Teshima; teshima63@nvlu.ac.jp

Received 3 September 2020; Revised 30 September 2020; Accepted 3 October 2020; Published 19 October 2020

Academic Editor: Quan Yuan

Copyright (C) 2020 Takahiro Teshima et al. This is an open access article distributed under the Creative Commons Attribution License, which permits unrestricted use, distribution, and reproduction in any medium, provided the original work is properly cited.

\begin{abstract}
The potential of mesenchymal stem cells (MSCs) to differentiate into nonmesodermal cells such as pancreatic beta cells has been reported. New cell-based therapy using MSCs for diabetes mellitus is anticipated as an alternative treatment option to insulin injection or islet transplantation in both human and veterinary medicine. Several protocols were reported for differentiation of MSCs into insulin-producing cells (IPCs), but no studies have reported IPCs generated from canine MSCs. The purpose of this study was to generate IPCs from canine adipose tissue-derived MSCs (AT-MSCs) in vitro and to investigate the effects of IPC transplantation on diabetic mice in vivo. Culturing AT-MSCs with the differentiation protocol under a two-dimensional culture system did not produce IPCs. However, spheroid-like small clusters consisting of canine AT-MSCs and human recombinant peptide $\mu$-pieces developed under a three-dimensional (3D) culture system were successfully differentiated into IPCs. The generated IPCs under 3D culture condition were stained with dithizone and anti-insulin antibody. Canine IPCs also showed gene expression typical for pancreatic beta cells and increased insulin secretion in response to glucose stimulation. The blood glucose levels in streptozotocin-induced diabetic mice were decreased after injection with the supernatant of canine IPCs, but the hyperglycemic states of diabetic mice were not improved after transplanting IPCs subcutaneously or intramesenterically. The histological examination showed that the transplanted small clusters of IPCs were successfully engrafted to the mice and included cells positive for insulin by immunofluorescence. Several factors, such as the transplanted cell number, the origin of AT-MSCs, and the differentiation protocol, were considered potential reasons for the inability to improve the hyperglycemic state after IPC transplantation. These findings suggest that canine AT-MSCs can be differentiated into IPCs under a 3D culture system and IPC transplantation may be a new treatment option for dogs with diabetes mellitus.
\end{abstract}

\section{Introduction}

Diabetes mellitus (DM) is a global concern not only in human medicine but also in veterinary medicine. DM is a major endocrine disease in dogs, and dogs with DM show similarities to patients with type $1 \mathrm{DM}$, which is classified as insulin- dependent diabetes. Owners of dogs with DM need to inject dogs with insulin every day. In humans, islet transplantation is one of the therapeutic options for type $1 \mathrm{DM}[1,2]$. However, islet transplantation has several limitations for widespread clinical use even in human medicine $[3,4]$ and is unfeasible in veterinary medicine. The field of regenerative 
medicine has progressed, and many researchers are aiming to establish new cell-based therapies for various diseases [5-7]. Transplantation of insulin-producing cells (IPCs) differentiated from stem cells is anticipated as an alternative to insulin injection or islet transplantation as a treatment option for DM patients. Several studies have reported the successful differentiation of pluripotent stem cells such as induced pluripotent stem cells (iPS) and embryonic stem cells (ES) into IPCs [8-11]. Some groups have attempted to differentiate multipotent stem cells such as mesenchymal stem cells (MSCs) into IPCs, and some results for potential cell-based therapies for DM have been reported [12-14]. Adipose tissue-derived MSCs (AT-MSCs) are an ideal source for IPC transplantation for veterinary medicine, because adipose tissue is abundant and can be obtained with minimally invasive procedures. The aim of this study was to perform differentiation of canine AT-MSCs into IPCs and investigate the effects of IPC transplantation on diabetic mice.

\section{Materials and Methods}

This research is composed of two studies. Study 1 performed the generation of IPCs from canine AT-MSCs in vitro. Study 2 conducted a functional evaluation of the canine IPCs on streptozotocin-induced diabetic mice. All experimental protocols involving the use of dogs and mice were approved by the Bioethics Committee at Nippon Veterinary and Life Science University.

2.1. Isolation and Expansion of AT-MSCs. Adipose tissue was aseptically collected from falciform ligament fat of three anaesthetized dogs (males; mean age, 1.5 years; mean body weight, $10.4 \mathrm{~kg}$ ). The tissue was washed extensively in phosphate buffer solution (PBS), minced, and digested with collagenase type I (Sigma-Aldrich) at $37^{\circ} \mathrm{C}$ for $45 \mathrm{~min}$ with intermittent shaking. After washing with PBS and centrifuging, the pellets containing the stromal vascular fraction were resuspended, filtered through a $100 \mu \mathrm{m}$ nylon mesh, and incubated overnight in Dulbecco's modified Eagle's medium (DMEM) supplemented with 10\% fetal bovine serum (FBS; Nichirei Bioscience) and a $1 \%$ antibiotic-antimycotic solution (Thermo Fisher Scientific) in a humidified atmosphere with $5 \% \mathrm{CO}_{2}$ at $37^{\circ} \mathrm{C}$. Unattached cells were removed by changing the medium, and the attached cells were washed twice with PBS. Thereafter, the medium was replaced every 3-4 days. At $80-90 \%$ confluence, the cells were detached with trypsinEDTA solution (Sigma-Aldrich) and passaged repeatedly.

2.2. Phenotype Analysis of Canine AT-MSCs. Passage 2 ATMSCs were analyzed by flow cytometry [15, 16]. Cells $\left(2 \times 10^{5}\right.$ cells $)$ were placed in fluorescence-activated cell sorting (FACS) tubes (BD Biosciences), washed with FACS buffer (PBS containing 2\% FBS), and then incubated with the following fluorescein- (FITC-) or phycoerythrin- (PE-) conjugated antibodies: anti-CD14-FITC (BD Pharmingen), antiCD29-PE (BioLegend), anti-CD34-PE (R\&D Systems), anti-CD44-PE (BioLegend), anti-CD45-FITC (eBioscience), and anti-CD90-PE (eBioscience) or their respective isotype controls. The cells were washed twice with FACS buffer and resuspended in $500 \mu \mathrm{l}$ of FACS buffer. Fluorescence was evaluated by flow cytometry in a FACSCalibur instrument (BD Biosciences). Data were analyzed using WinMDI 2.9 analysis software.

2.3. Differentiation of Canine AT-MSCs into IPCs. Differentiation was performed according to a previously reported protocol [17], with modifications. Canine AT-MSCs at passage 3 were seeded in conventional 6-well attachment plates $\left(1.5 \times 10^{6}\right.$ cells/well $)$ or 96 -well ultralow attachment plates (Thermo Fisher Scientific) with or without $\mu$-pieces (cellnest $\mu$-piece, Fujifilm $)\left(1.0 \times 10^{5}\right.$ cells/well with or without $\mu$-pieces of $0.05 \mathrm{mg} /$ well) as 3D culture. AT-MSCs were cultured using step 1 (from day 0 to day 2) medium containing FBS-free DMEM with high glucose $(25 \mathrm{mM})$ and $0.5 \mathrm{mM} \beta$-mercaptoethanol (Sigma-Aldrich). At day 3, small clusters that formed in the 96-well ultralow attachment plates were transferred into 6-well ultralow attachment plates (Corning). Next, cells were cultured in step 2 (from day 3 to day 10) medium containing FBS-free DMEM with high glucose (25 mM), 1\% glutamine supplement (GlutaMAX, Thermo Fisher Scientific), $1 \%$ nonessential amino acid (FUJIFILM Wako), $20 \mathrm{ng} / \mathrm{ml}$ of recombinant human basic fibroblast growth factor (FUJIFILM Wako), $20 \mathrm{ng} / \mathrm{ml}$ of recombinant human epidermal growth factor (FUJIFILM Wako), 1\% N2 supplement (Thermo Fisher Scientific), 1\% B27 supplement (Thermo Fisher Scientific), and 10 nM exendin-4 (Sigma-Aldrich). Finally, cells were cultured in step 3 (from day 11 to day 21) medium containing FBS-free DMEM with high glucose (25 mM), 1\% N2 supplement, 1\% B27 supplement, $10 \mathrm{nM}$ exendin-4, $10 \mathrm{mM}$ nicotinamide (FUJIFILM Wako), $10 \mathrm{ng} / \mathrm{ml}$ betacellulin (Sigma-Aldrich), $50 \mathrm{ng} / \mathrm{ml} \mathrm{recombinant}$ human activin-A (FUJIFILM Wako), and $50 \mathrm{ng} / \mathrm{ml}$ recombinant human hepatocyte growth factor (FUJIFILM Wako).

2.4. Dithizone (DTZ) Staining. DTZ (Sigma-Aldrich) staining was performed according to a previously reported protocol $[17,18]$. DTZ stock solution was prepared with $50 \mathrm{mg}$ of DTZ in $5 \mathrm{ml}$ of dimethyl sulfoxide and stored at $-20^{\circ} \mathrm{C}$. For staining, $10 \mu \mathrm{l}$ of the stock solution was added to $1 \mathrm{ml}$ of serum-free DMEM and filtered through a $0.2 \mu \mathrm{m}$ nylon filter; this solution was used as the DTZ working solution. Cells at the end of the differentiation protocol were incubated in the DTZ working solution at $37^{\circ} \mathrm{C}$ for $30 \mathrm{~min}$. Cells were examined with a stereomicroscope after the plates were rinsed three times with PBS.

2.5. Quantitative RT-PCR. Total RNA from differentiated cells was extracted using a TRIzol Plus RNA Purification Kit according to the manufacturer's instructions (Thermo Fisher Scientific). cDNA was synthesized from $1 \mu \mathrm{g}$ of total RNA using random primers and the GoScript Reverse Transcriptase System (Promega), according to the manufacturer's instructions. Real-time RT-PCR analyses were performed using the SYBR Green Real-Time PCR Master Mix (Promega) to determine the mRNA levels of pancreatic duodenal homeobox (Pdx-1), neurogenin 3 (Ngn3), and glucose transporter 2 (GLUT2). Glyceraldehyde 3-phosphate dehydrogenase (GAPDH) mRNA level was used for normalization. 
The primer sequences were as follows: Pdx-1-forward $5^{\prime}$ -CCTACGTTGCAGAGCCAGAA-3 ${ }^{\prime}$ and reverse $5^{\prime}$-GTGC CTCTCGGTCAAGTTCA-3'; Ngn3-forward $5^{\prime}$-GAGC GCAATCGAATGCACAA- $3^{\prime}$ and reverse $5^{\prime}$-TAGAGG CTGTGGTCCGCTAT-3'; GLUT2-forward $5^{\prime}$-CCCACA ACCCCATGCCTAAT- $3^{\prime}$ and reverse $5^{\prime}$-ATCCCCTAGCC ATCCACCAA-3 ${ }^{\prime}$; and GAPDH-forward $5^{\prime}$-GATGGG CGTGAACCATGAG- $3^{\prime}$ and reverse $5^{\prime}$-TCATGAGGCCC TCCACGAT- $3^{\prime}$. Amplification conditions were $95^{\circ} \mathrm{C}$ for $2 \mathrm{~min}$, followed by 40 cycles of $95^{\circ} \mathrm{C}$ for $15 \mathrm{sec}$ and $60^{\circ} \mathrm{C}$ for $60 \mathrm{sec}$. After 40 cycles, a dissociation curve was generated to verify the specificity of each primer. All reactions were performed in duplicate. Expression levels of target genes were normalized to the level of GAPDH and quantified by the $\Delta \Delta \mathrm{Ct}$ method.

2.6. Glucose-Stimulated Insulin Secretion Assay. To determine the in vitro ability of the differentiated AT-MSCs, the insulin secretory response to glucose was measured using a previously reported method $[12,17,19]$, with modifications. The differentiated cells $\left(1.5 \times 10^{6}\right.$ AT-MSCs $)$ were preincubated for $1 \mathrm{~h}$ in glucose-free Krebs-Ringer bicarbonate (KRB). The cells were then incubated with KRB containing $5.5 \mathrm{mM}$ or $25 \mathrm{mM}$ of glucose for $2 \mathrm{~h}$. The KRB media were collected and frozen at $-80^{\circ} \mathrm{C}$ until evaluation. The insulin level was measured using a canine insulin ELISA kit (FUJIFILM Wako Shibayagi).

2.7. Immunofluorescence. The small clusters including $\mu$ pieces formed from the $3 \mathrm{D}$ culture system (study 1) and transplanted IPCs (study 2 ) were fixed in $4 \%$ paraformaldehyde, dehydrated, and embedded in paraffin; samples were cut into $4 \mu \mathrm{m}$ sections. The sections were dewaxed in xylene, hydrated, and blocked with a commercial blocking reagent (UltraCruz Blocking Reagent, Santa Cruz) for $1 \mathrm{~h}$ in a humidified chamber at room temperature. Then, sections were incubated with a monoclonal insulin antibody conjugated with Alexa Flour 488 (1:100 dilution; 2D11-H5, Santa Cruz) overnight at $4^{\circ} \mathrm{C}$ in a humidified chamber. After washing with PBS containing $0.05 \%$ of Tween 20 , sections were covered with mounting medium with DAPI (VECTASHIELD, H-1200, Vector Laboratories). Immunofluorescence images were captured using a multipurpose microscope (BZX700, Keyence).

2.8. STZ-Induced Diabetic Mice. Twenty-five male C57BL/6JJcl mice (B6, 5-6 weeks) and fifteen male immunodeficient mice (Crlj:SHO-Prkdc scid $\mathrm{Hr}^{h r}$; SHO, 5-6 weeks) were used in study 2 . The mice were housed in a temperaturecontrolled and light-controlled room ( $12 \mathrm{~h}$ light/dark cycles) and allowed free access to water and standard laboratory food.

To induce a diabetic state in the mice, $200 \mathrm{mg} / \mathrm{kg}$ of streptozotocin (STZ, Sigma-Aldrich) was administrated intraperitoneally as a single injection. The diabetic states were confirmed after two consecutive measurements of blood glucose level over $350 \mathrm{mg} / \mathrm{dl}$ or one measurement over $400 \mathrm{mg} / \mathrm{dl}$ with a glucometer.
2.9. Effect of Insulin Secreted from Canine IPC on STZInduced Diabetic B6 Mice. Twenty-one B6 mice confirmed with the diabetic status were divided into four treatment groups: group I: $200 \mu \mathrm{l}$ of PBS $(n=5)$, group II: $200 \mu \mathrm{l}$ of FBS-free DMEM ( $n=5)$, group III: $200 \mu$ l of the supernatant of undifferentiated AT-MSC culture medium $(n=5)$, and group IV: $200 \mu \mathrm{l}$ of the supernatant of canine IPC culture medium (containing $1 \mathrm{ng}$ of canine insulin as measured by ELISA) $(n=6)$. All mice were kept in fasting condition (with free access to water) for $12 \mathrm{~h}$ after intraperitoneal injection with the appropriate solution, and blood glucose levels were measured at $0,3,6,9$, and $12 \mathrm{~h}$ after injection.

The blood glucose levels of group IV mice were reevaluated as the same time points with alternative injection with $200 \mu \mathrm{l}$ of FBS-free DMEM intraperitoneally after 2 days from administration of the supernatant of IPC culture medium.

2.10. IPC Transplantation to STZ-Induced Diabetic SHO Mice. Twelve mice confirmed with diabetic status were divided into four groups ( $n=3$ mice/group): group I (control): intraperitoneally injected with PBS, group II: intraperitoneally injected with $1.5 \times 10^{6}$ undifferentiated AT-MSCs, group III: transplanted with small clusters of IPCs into the inguinal subcutaneous region, and group IV: transplanted with small clusters of IPCs into the intramesentery. In groups III and IV, mice were transplanted with small clusters of IPC formed from 3D culture with $\mu$-pieces (30 wells of 96-well ultralow attachment plates) under anesthesia with a mixture of medetomidine $(0.75 \mathrm{mg} / \mathrm{kg})$, midazolam $(4 \mathrm{mg} / \mathrm{kg})$, and butorphanol $(5 \mathrm{mg} / \mathrm{kg})$. Nonfasting blood glucose levels were measured at days $3,7,10$, and 15 after injection or transplantation. Transplanted mice were sacrificed at day 15 after blood collection for measurement of canine insulin concentrations, and the transplanted IPC clusters were harvested for histological analysis.

2.11. Statistical Analysis. All data are presented as the mean \pm standard deviation. Differences between two groups were compared with the Welch $t$-test. Differences among multiple groups were assessed by one-way or two-way analysis of variance, and differences were compared using the TukeyKramer post hoc test. $P<0.05$ was considered statistically significant. Statistical analyses were performed using Excel 2019 with add-in software Statcel 3.

\section{Results}

3.1. Characterization of AT-MSCs. AT-MSCs from three beagles were successfully cultured and expanded as described in Materials and Methods. The majority of the cells expressed the mesenchymal stem cell markers CD29 (97.58 $\pm 1.14 \%)$, CD44 (99.54 $\pm 0.25 \%)$, and CD90 (96.23 $\pm 0.97 \%)$, and very few expressed CD14 (1.38 $\pm 0.08 \%)$, CD34 (0.68 $\pm 0.06 \%)$, or CD45 $(1.13 \pm 0.08 \%)$.

\subsection{Generation of IPCs from Canine AT-MSCs In Vitro (Study 1)}

3.2.1. DTZ Staining. Differentiated AT-MSCs under conventional culture conditions (cultured in attachment dish) 
Unstained

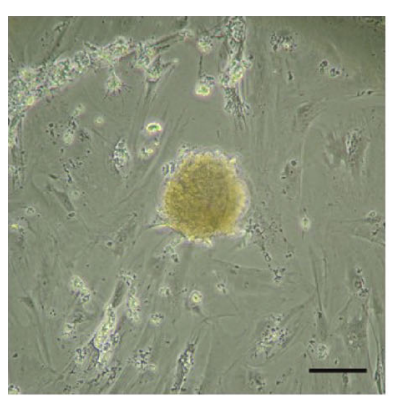

(a)
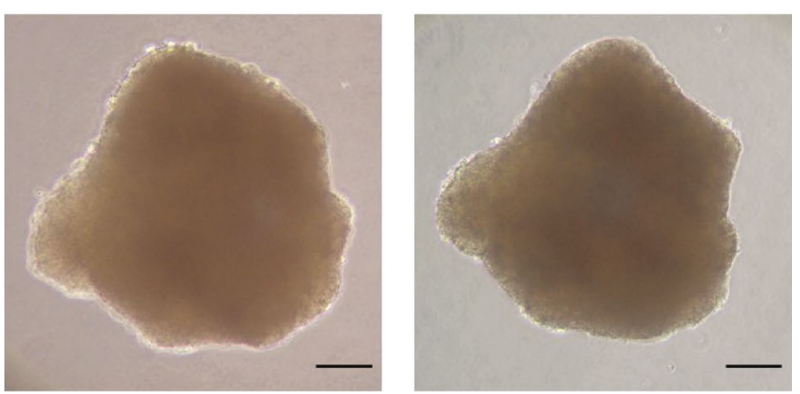

(b)
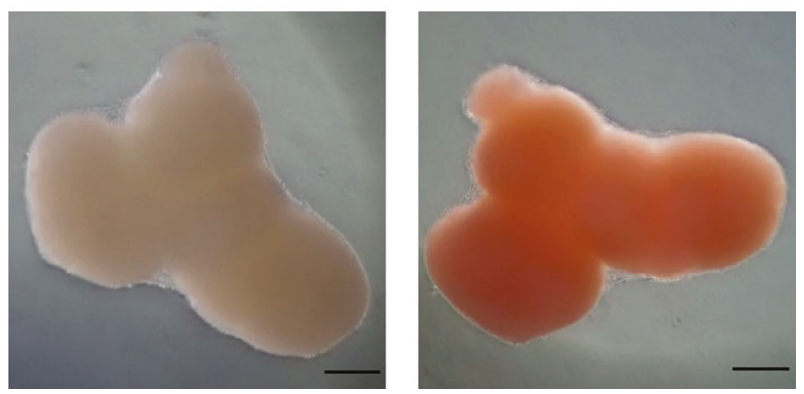

(c)

FIGURE 1: Dithizone staining of canine adipose tissue-derived mesenchymal stem cells at the end of in vitro differentiation. (a) Cells cultured in the differentiated protocol using the twodimensional culture system using an attachment plate. (b) Undifferentiated adipose tissue-derived mesenchymal stem cells using the three-dimensional culture system with $\mu$-pieces. (c) Differentiated adipose tissue-derived mesenchymal stem cells using the three-dimensional culture system with $\mu$-pieces. Bar $=$ $200 \mu \mathrm{m}$.

were not stained with DTZ, while differentiated AT-MSCs cultured under 3D conditions with or without $\mu$-pieces were stained with DTZ (Figure 1).

3.2.2. Gene Expression. To clarify the characteristic features of the differentiated AT-MSCs cultured under 3D condition with $\mu$-pieces, the gene expression of endocrine pancreatic markers was assessed using quantitative RT-PCR. The expressions of Pdx-1, Ngn3, and GLUT2 mRNAs were significantly higher in differentiated AT-MSCs under 3D condition with $\mu$-pieces compared with levels in undifferentiated AT-MSCs $(P<0.05)$ (Figure 2$)$.
3.2.3. Glucose-Stimulated Insulin Secretion. We next evaluated the insulin secretion from canine IPC in response to glucose (Figure 3). Low levels of insulin were detected in the supernatant of differentiated AT-MSCs cultured under 2D condition when stimulated with high glucose. Otherwise, the differentiated AT-MSCs under 3D condition with or without $\mu$-pieces secreted insulin when stimulated with low glucose (with $\mu$-pieces, $1.18 \pm 0.10 \mathrm{ng} / \mathrm{ml}$; without $\mu$-pieces, $0.89 \pm 0.10 \mathrm{ng} / \mathrm{ml}$ ). The differentiated AT-MSCs under 3D condition with $\mu$-pieces secreted significantly higher insulin levels compared with cells cultured without $\mu$-pieces $(P<0.05)$. Differentiated AT-MSCs cultured under 3D condition with and without $\mu$-pieces secreted higher amounts of insulin in response to high glucose concentrations compared with low glucose (with $\mu$-pieces, $1.88 \pm 0.17 \mathrm{ng} / \mathrm{ml}$; without $\mu$-pieces, $1.24 \pm 0.14 \mathrm{ng} / \mathrm{ml}$ ).

3.2.4. Immunofluorescence Staining. Immunofluorescence analysis was performed to investigate the expression of insulin in AT-MSCs. Differentiated AT-MSCs under 3D condition showed positive cytoplasmic staining for insulin, while undifferentiated AT-MSCs were negative for insulin (Figure 4).

\subsection{Functional Assessment of the IPCs from Canine AT- MSCs In Vivo (Study 2)}

3.3.1. Effect of Insulin Secreted from Canine IPC on STZInduced Diabetic B6 Mice. STZ-induced diabetic mice were kept in fasting condition after intraperitoneal injection with each solution. The blood glucose levels of groups I (PBS), II (DMEM), and III (supernatant of ADSC culture medium) gradually decreased after $12 \mathrm{~h}$ observation (group I, $433 \mathrm{mg} / \mathrm{dl}$ to $322 \mathrm{mg} / \mathrm{dl}$; group II, $473 \mathrm{mg} / \mathrm{dl}$ to $303 \mathrm{mg} / \mathrm{dl}$; and group III, $527 \mathrm{mg} / \mathrm{dl}$ to $343 \mathrm{mg} / \mathrm{dl}$ ). In contrast, the blood glucose levels of group IV (supernatant of IPC culture medium) at $0 \mathrm{~h}$ were $472 \mathrm{mg} / \mathrm{dl}$, but after administration of the supernatant of IPCs, the levels significantly decreased compared with those of groups I, II, and III; levels at 3, 6, 9, and $12 \mathrm{~h}$ were $336,322,200$, and $123 \mathrm{mg} / \mathrm{dl}$, respectively $(P<0.05)$ (Figure 5(a)).

Group IV mice were injected with $200 \mu$ l of FBS-free DMEM, and blood glucose levels were observed during $12 \mathrm{~h}$ after 2-day administration of the supernatant of IPCs. The blood glucose concentrations of all mice were not remarkably decreased after injection with DMEM (Figure 5(b)).

\subsubsection{IPC Transplantation to STZ-Induced Diabetic SHO}

Mice. Blood glucose concentrations were monitored during 15 days after IPC subcutaneous or intramesenteric transplantation. All mice of groups II (undifferentiated AT-MSC transplantation), III (subcutaneous transplantation), and IV (intramesenteric transplantation) were alive over the 15 days in the observation period, but all three mice in group I (control) died at day $7(n=1)$ and day $13(n=2)$. The blood glucose level of the mouse in group III was decreased to $171 \mathrm{mg} / \mathrm{dl}$ at day 15; however, both group III and IV mice had a continued hyperglycemic state after IPC transplantation (mean blood glucose levels at day 15 were $546 \mathrm{mg} / \mathrm{dl}$, $320 \mathrm{mg} / \mathrm{dl}$, and $359 \mathrm{mg} / \mathrm{dl}$ in groups II, III, and IV, 

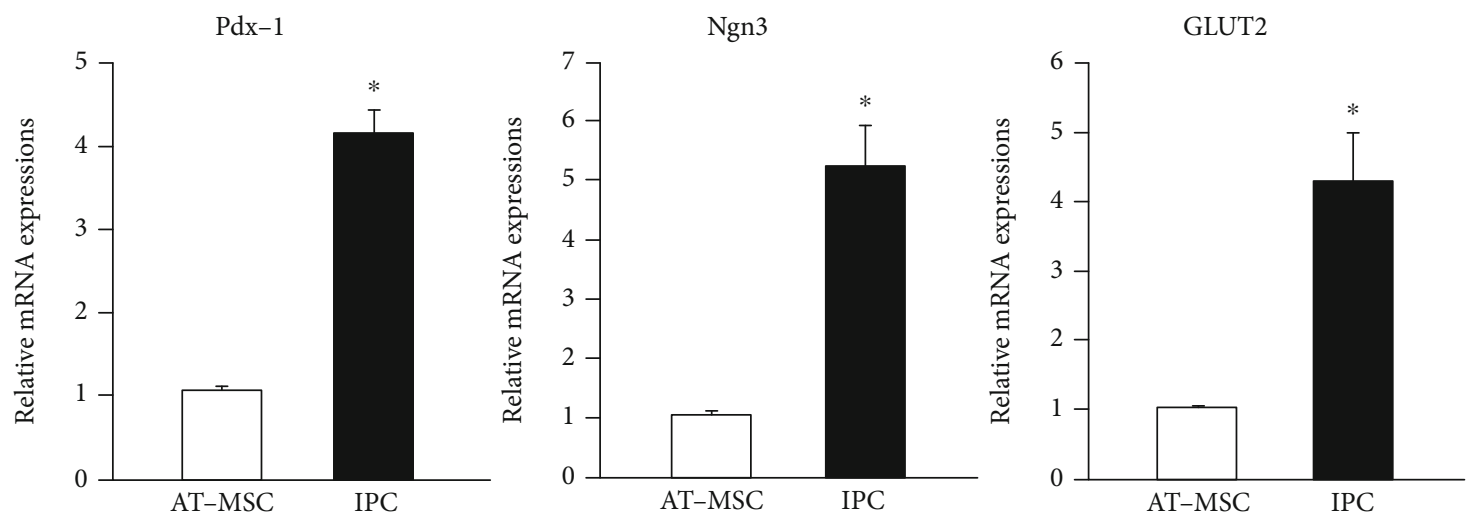

FIGURE 2: Expression of endocrine pancreatic marker genes in undifferentiated and differentiated adipose tissue-derived mesenchymal stem cells. Pdx-1, Ngn3, and GLUT2 mRNA expressions in insulin-producing cells (IPC) generated under the three-dimensional culture system with $\mu$-pieces were $4.1,5.2$, and 4.3 times higher, respectively, than those in undifferentiated adipose tissue-derived mesenchymal stem cells (AT-MSCs). Data are expressed as the mean \pm standard deviation. ${ }^{*} P<0.05$ vs. AT-MSC.

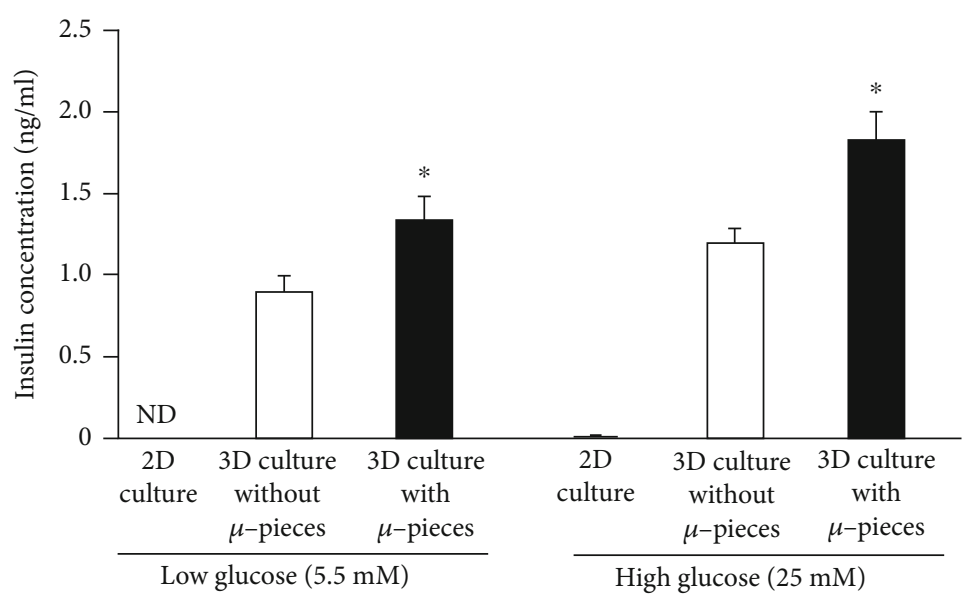

FIGURE 3: In vitro insulin release in response to glucose stimulation. The insulin secretion ability under glucose stimulation was higher in the IPCs generated by the 3 -dimensional (3D) culture system with $\mu$-pieces than in cells cultured without $\mu$-pieces. Data are expressed as the mean \pm standard deviation. ${ }^{*} P<0.05$ vs. $3 \mathrm{D}$ culture without $\mu$-pieces. ND: not detected.
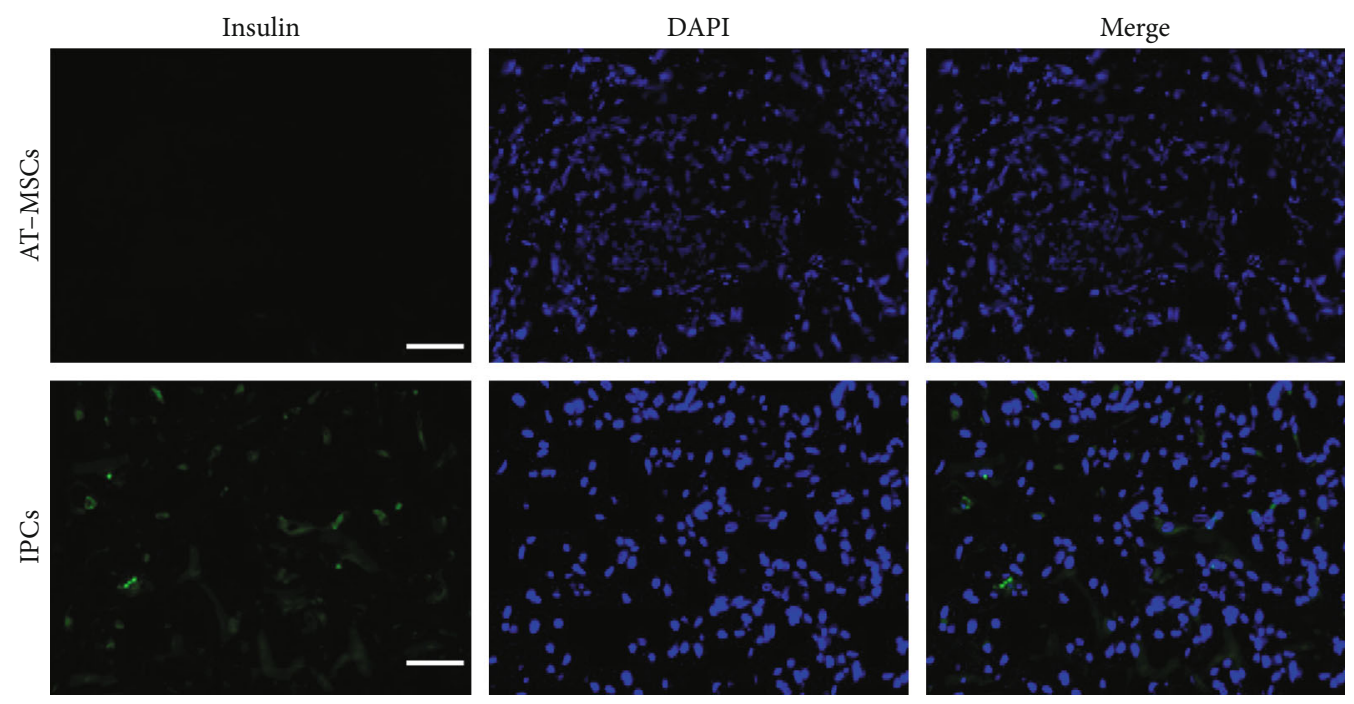

FIGURE 4: Immunofluorescence of canine adipose-derived mesenchymal stem cells (AT-MSCs) following in vitro differentiation into insulinproducing cells (IPCs). Cells were stained for insulin (green) with counterstaining for DAPI (blue). Bar $=50 \mu \mathrm{m}$. 


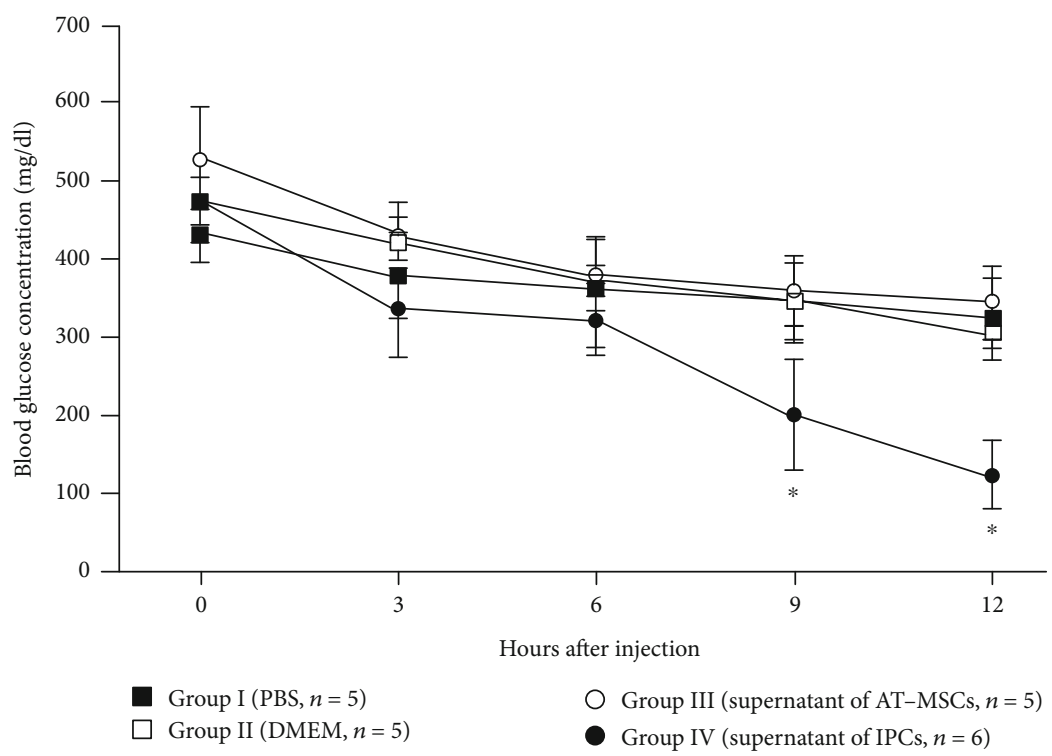

(a)
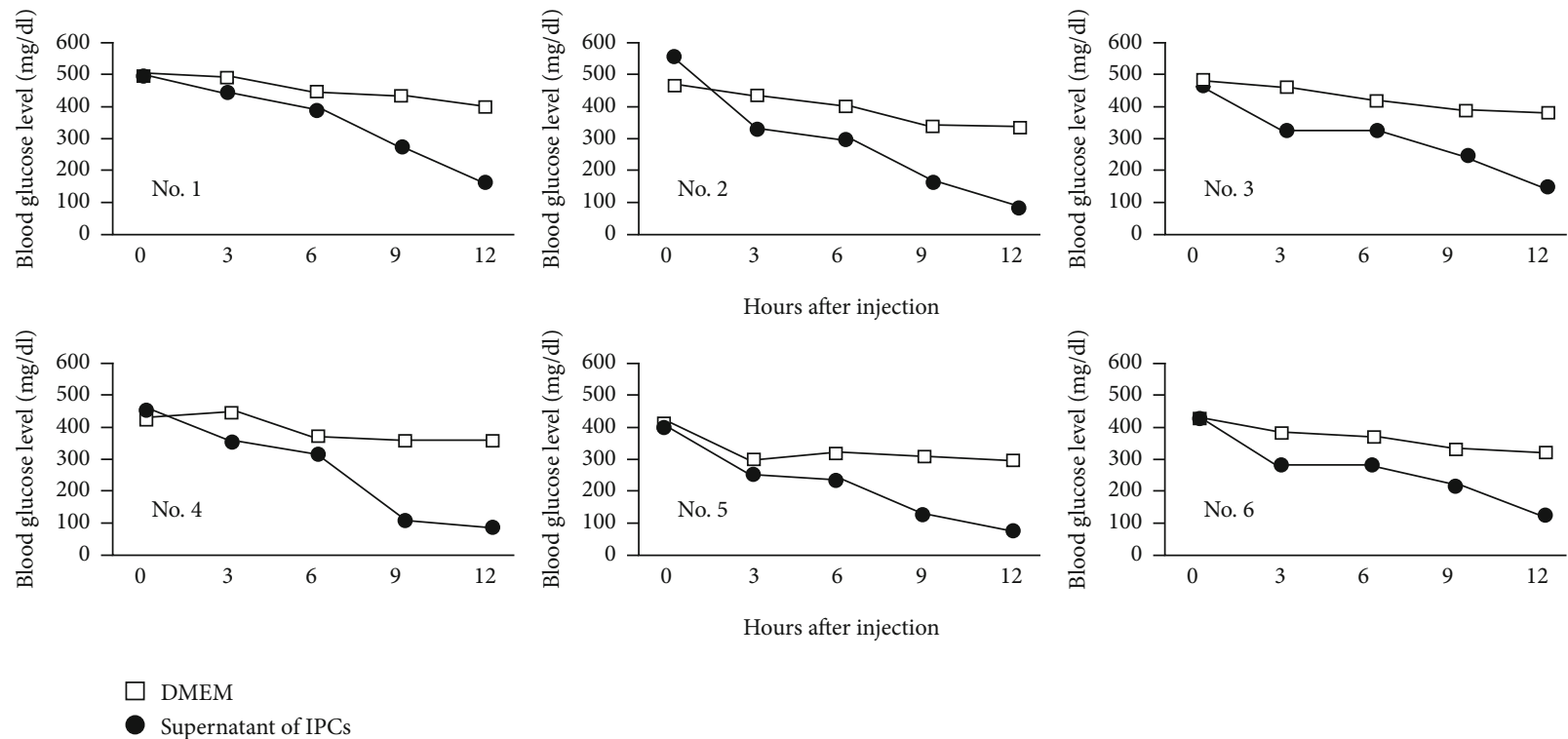

- Supernatant of IPCs

(b)

Figure 5: Blood glucose levels in diabetic mice after injection with various treatments. (a) Comparison among groups. The blood glucose levels at 9 and $12 \mathrm{~h}$ after injection in group IV (supernatant of IPCs) were significantly lower compared with those in groups I (PBS), II (DMEM), and III (supernatant of AT-MSCs). Data are expressed as the mean \pm standard deviation. ${ }^{*} P<0.05$ vs. groups I, II, and III. (b) Comparison of the blood glucose levels in the same mice after injection with the supernatant of IPCs and DMEM. The blood glucose levels were lower after injection with the supernatant of IPCs in all six mice.

respectively) (Figure 6). Serum canine insulin concentrations in all groups at day 15 were not detected by a canine insulin ELISA.

3.3.3. Histopathology of the Transplanted IPCs. We next examined the transplanted IPCs at subcutaneous and intramesentery sites on day 15 posttransplantation. Blood vessels were present at the surface of IPC clusters, and IPC clusters seemed to be successfully engrafted at transplanted sites. Light microscopic imaging showed vascularization of inner IPC clusters, and central necrosis of IPCs was not observed
(Figure 7). Both subcutaneous and intramesenteric transplanted IPC clusters showed positive insulin staining by immunofluorescence (Figure 8).

\section{Discussion}

Adipose tissue is an abundant source of MSCs, and ATMSCs can differentiate toward adipogenic, chondrogenic, and osteogenic cell lineages. Recent studies showed that AT-MSCs have the potential to differentiate into nonmesodermal cells such as neural cells, hepatocytes, and pancreatic 


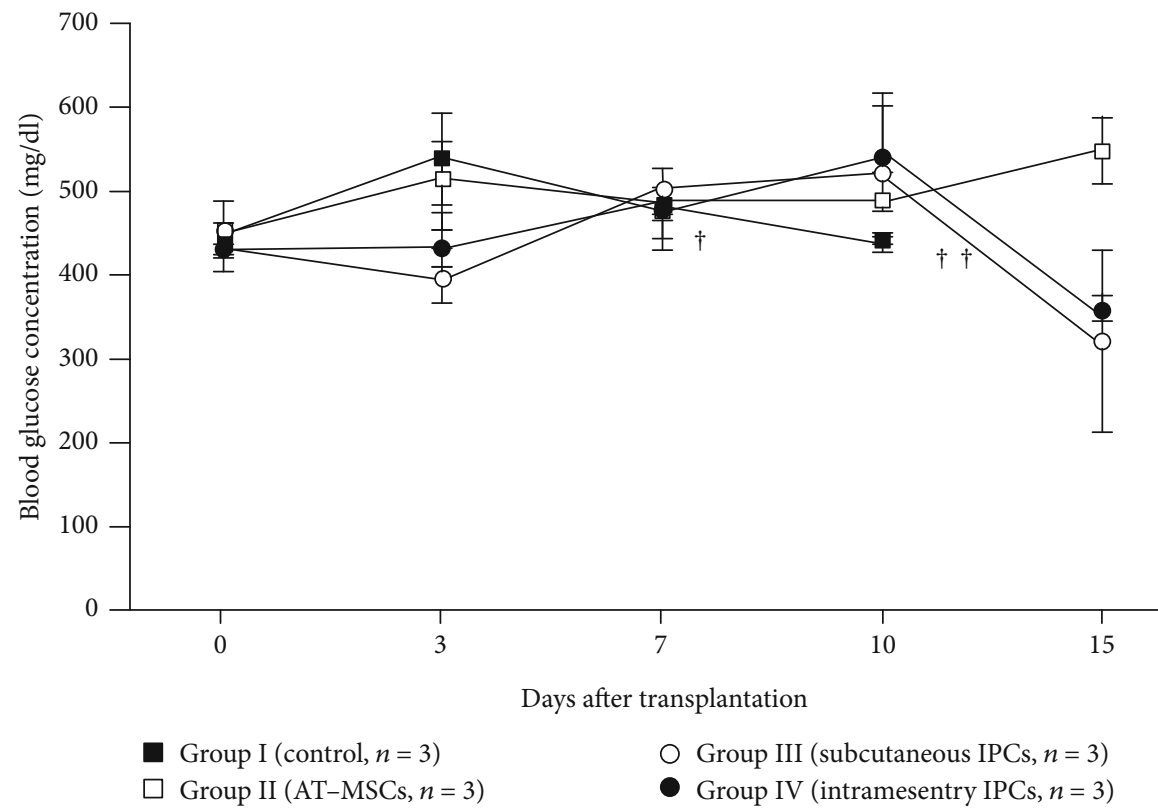

Figure 6: Nonfasting blood glucose levels after transplantation. The blood glucose levels at day 15 were decreased in groups III (subcutaneous IPC transplantation) and IV (intramesentery IPC transplantation) compared with group II (AT-MSC transplantation), but the hyperglycemic state was not improved. $\uparrow$ : death in group IV.

beta cells [20-24]. Therefore, new strategies for the treatment of DM using AT-MSCs that differentiated into IPCs have been actively researched $[25,26]$. In the veterinary field, canine DM is a common endocrine disease, and currently, the only available treatment option for canine DM is insulin injection throughout life.

To establish a new treatment option for canine DM as an alternative to daily insulin injection, we performed differentiation of canine AT-MSCs into IPCs in vitro (study 1) and examined the function of the generated IPCs from canine AT-MSCs in vivo (study 2).

Several protocols for differentiating cells into IPCs have been reported in iPS, ES, and MSCs. In this study, we performed a method similar to the previously reported method using human BM-MSCs [17], with modifications. This protocol involves three steps. In the first step, $\beta$-mercaptoethanol was used to induce the expression of the transcription factor Pdx-1, also known as insulin promoter factor 1. Pdx1 is required for the early embryonic development of the pancreas and is also required for the subsequent differentiation [27]. The next step involves EGF and bFGF, which play an important role in cellular proliferation, differentiation, and survival [28]. EGF and bFGF have been reported to be useful in IPC differentiation $[29,30]$. Exendin- 4 can boost the expression of beta cell-related genes Pdx-1, Nkx2.2, Isl-1, and MafA [31]. Furthermore, IPCs generated from Wharton's jelly MSCs using a differentiated protocol with exendin- 4 showed much better response to variable glucose levels [31]. The third step involves activin-A, betacellulin, nicotinamide, and hepatocyte growth factor. Many studies have used these substances to differentiate cells into IPCs in vitro. The combination of activin- $\mathrm{A}$ and betacellulin has been reported to enhance the proliferation into IPCs [32, 33]. Nicotinamide, which is a poly-ADP-ribose polymerase inhibitor, functions in the preservation of islet viability and function and represents a common extrinsic induction factor for MSCs [34]. HGF has been suggested to be a potent regulator of beta cell function and proliferation, and the effects of HGF are enhanced by activin-A $[35,36]$. High glucosecontaining medium was used throughout the differentiation protocol, because high glucose levels have been considered a potent inducer for pancreatic islet differentiation [29].

In our protocol for IPC differentiation, $\mu$-pieces were used for three-dimensional cell culture. Several 3D culture materials have been established, such as Matrigel, scaffolds, and unattached culture dishes [37-39]. 3D cell culture supports tissue-specific function and physiological cell-cell and cell-matrix interactions. These factors contribute to various advantages for increasing cell viability, improving cell-type specific function and gene expression, and increasing cell secretion of proteins [40]. The advantage of $\mu$-pieces, such as human recombinant peptide petaloid, was reported for islet cell culture and cell transplantation [41]. Spheroid-like cell aggregation-formed combination cells with $\mu$-pieces can prevent central necrosis within cell aggregates, because nutrients and waste products can pass through the inner spheroid [42]. Our results showed that IPC generated from canine AT-MSCs in the 3D culture system showed better DTZ staining and insulin secretion compared with cells in conventional $2 \mathrm{D}$ culture dishes. These results were in agreement with a previous study that demonstrated that the $3 \mathrm{D}$ culture system using a collagen/hyaluronic acid scaffold enhanced the differentiation of IPCs from rat AT-MSCs [14]. Our findings demonstrated that canine AT-MSCs can be differentiated into IPCs using the protocol modified from human BM-MSCs under a 3D culture system.

We next performed functional evaluation of the IPCs from canine AT-MSCs in vivo. We first confirmed the 

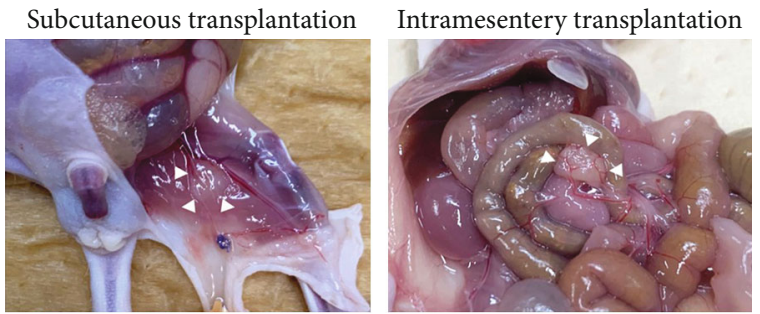

(a)
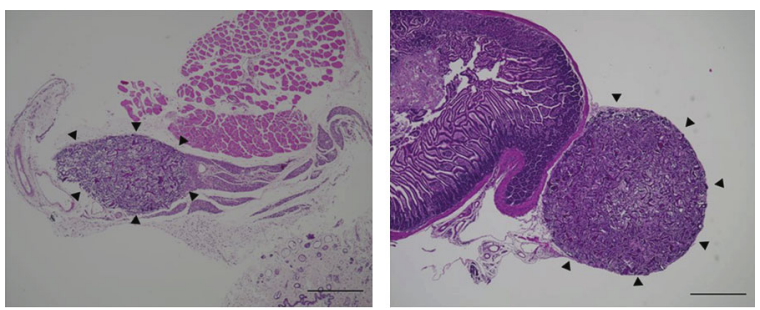

(b)
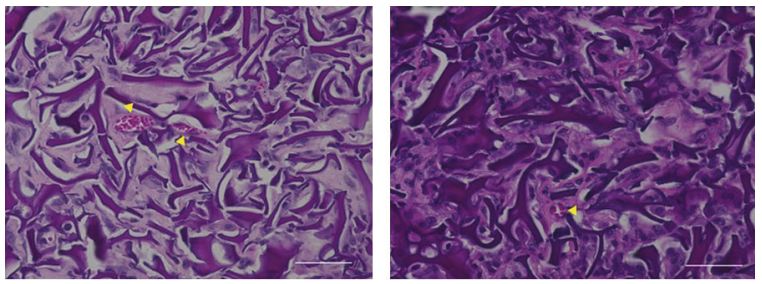

(c)

Figure 7: Transplanted small clusters of IPCs at subcutaneous and intramesentery sites. (a) Images at autopsy. The microvessels ran through the surface of both subcutaneous and intramesentery transplanted small clusters of IPCs (white arrowheads). IPCs engrafted in the transplanted sites in the mice. (b) Hematoxylineosin (HE) staining in the transplanted small clusters of IPCs (black arrowheads). Bar $=500 \mu \mathrm{m}$. (c) Necrosis in the central location of small clusters of IPCs was not observed, but angiogenesis (yellow arrowheads) was confirmed in HE staining. $\mathrm{Bar}=50 \mu \mathrm{m}$.

improvement of the hyperglycemic state in diabetic mice after administration of the supernatant of IPCs differentiated from canine AT-MSCs. The blood glucose levels of all six mice in group IV (treated with the supernatant of IPCs) were significantly decreased compared with those of other groups. In an additional examination, all mice in group IV did not show improvement of the hyperglycemic state after administration with culture medium. This result demonstrated that insulin produced by canine IPCs decreased the blood glucose levels in STZ-induced diabetic mice.

Next, we assessed the effects of transplanted canine IPCs on STZ-induced diabetic SHO mice. After 15 days of observation, microvessels were observed on the surface of transplanted small clusters of IPCs. Histological examination revealed that small clusters of IPCs were engrafted in transplanted sites and included insulin-positive cells as identified by immunofluorescence. However, IPCs transplanted subcutaneously or intramesenterically did not improve the hyperglycemic states. There are several possible reasons for the failure to improve the hyperglycemic status after IPC transplantation. The first possibility may be that the number of
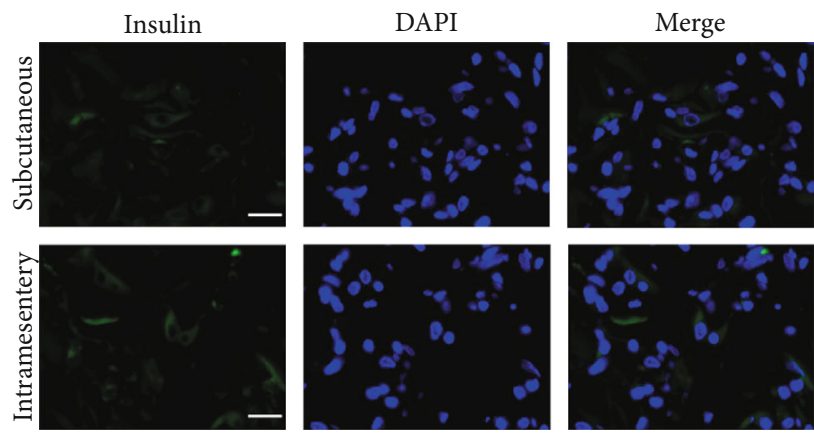

FIGURE 8: Immunofluorescence of transplanted small clusters of IPCs in diabetic mice. Small clusters of IPCs showed positive insulin staining (green) in both subcutaneous transplantation and intramesentery transplantation. Bar $=50 \mu \mathrm{m}$.

transplanted IPCs is not enough for the donor's body size. We transplanted $1.5 \times 10^{6}$ AT-MSCs according to a previous study that showed transplantation of IPCs differentiated from human BM-MSCs into STZ-induced diabetic rats [43]. The study demonstrated that intraperitoneal transplantation of $1.5 \times 10^{6} \mathrm{BM}-\mathrm{MSCs}$ that differentiated into IPCs into diabetic rats led to the improvement of the hyperglycemic states. However, in a recent report on the transplantation of IPCs generated from human AT-MSCs with $\mu$-pieces into diabetic mice, $4.8 \times 10^{7}$ cells were transplanted under the kidney capsule or intramesentery region and led to normoglycemic states $[44,45]$. One of these studies also calculated the cell number of generated IPCs and found that $1.8 \times 10^{4}$ IPCs were obtained from $5 \times 10^{5}$ AT-MSCs, indicating that $3.6 \%$ of the initial AT-MSCs differentiated into IPCs; thus, approximately $1.7 \times 10^{6}$ IPCs are needed per mouse to gain the normoglycemic states in STZ-induced diabetic mice [45]. In our study, we did not calculate the differentiation ratio of IPCs, but the differentiation protocol referred to in our study reported approximately that $2.5 \%$ of human BMMSCs differentiate into insulin-positive cells under a 2D conventional culture system [17]. These reports suggest that our cell numbers were too small to improve the hyperglycemic states in STZ-induced diabetic mice. Therefore, we will need to evaluate the quantitative differentiation efficiency in further study.

The second possible cause is the origin of the AT-MSCs. In our study, we collected adipose tissue from falciform ligament fat and isolated AT-MSCs. A previous study on the insulin secretion capacity of IPCs generated from human AT-MSCs reported that IPCs generated from visceral ATMSCs showed poor differentiation and insulin secretion compared with those from subcutaneous AT-MSCs [45]. One reason why visceral AT-MSCs have been not suitable for differentiation into IPCs is that visceral AT-MSCs secrete more inflammatory cytokines such as IL-6 and TNF- $\alpha$ [46]. Multiple signalling cascades stimulated by inflammatory cytokines activate inducible nitric oxide synthase and result in apoptosis with impaired insulin biosynthesis and secretion. The study also indicated that IPCs generated from visceral AT-MSCs were inferior regarding glucose-stimulated insulin secretion. A stimulation index (SI), a parameter that 
reflects the insulin releasing function in response to glucose levels, of less than 3 does not normalize blood glucose levels after transplantation [45]. Our generated IPCs from canine visceral AT-MSCs showed increasing insulin secretion in response to high glucose levels, but the SI of our generated IPCs in vitro was only approximately 1.4 (calculated as the level of insulin concentration under high glucose condition $(25 \mathrm{mM})$ divided by that under low glucose condition $(5.5 \mathrm{mM})$ ). Our result of glucose-stimulated insulin secretion ability was similar to that of human visceral IPCs (SI of subcutaneous IPCs was 3.8, but SI of visceral IPCs was 1.5) [45]. Therefore, our generated IPCs may not show sufficient insulin releasing function for improving the hyperglycemic state in vivo.

To differentiate cells into IPCs with more potent insulin secretion capacity, a more effective differentiation protocol will be needed. Other differentiation protocols containing histone deacetylase (HDAC) inhibitors such as trichostatin A and valproic acid have been reported [19, 47]. HDAC inhibitors induce a more open chromatin architecture and increased access for transcription factors. Moreover, HDAC inhibition was reported to be a potent driver of pancreatic cell lineage progenitors [48]. Therefore, we will need to evaluate other differentiation protocols to determine which protocol will be more suitable for canine AT-MSCs.

\section{Conclusion}

The results of this study demonstrated that the canine ATMSCs can be differentiated into IPCs under a 3D culture system. To achieve stem cell-based therapy for canine DM for clinical use, further studies are required to establish a more effective differentiation protocol that induces increased insulin secretion in vitro and to determine the number of transplanted cells that are sufficient to improve hyperglycemic states in vivo.

\section{Data Availability}

The data supporting the findings of this study are available from the corresponding author upon reasonable request.

\section{Conflicts of Interest}

The authors declare no conflict of interests.

\section{Acknowledgments}

We thank Gabrielle White Wolf, $\mathrm{PhD}$, from Edanz Group (https://en-author-services.edanzgroup.com/ac) for editing the draft of this manuscript.

\section{References}

[1] A. M. Shapiro, M. Pokrywczynska, and C. Ricordi, "Clinical pancreatic islet transplantation," Nature Reviews Endocrinology, vol. 13, no. 5, pp. 268-277, 2017.

[2] M. R. Rickels and R. P. Robertson, "Pancreatic islet transplantation in humans: recent progress and future directions," Endocrine Reviews, vol. 40, no. 2, pp. 631-668, 2019.
[3] E. A. Ryan, B. W. Paty, P. A. Senior et al., "Five-year follow-up after clinical islet transplantation," Diabetes, vol. 54, no. 7, pp. 2060-2069, 2005.

[4] J. S. Danobeitia and L. A. Fernandez, "Clinical islet transplantation: recent advances in the field," Clinical Transplantation, pp. 193-200, 2012.

[5] W. Zakrzewski, M. Dobrzyński, M. Szymonowicz, and Z. Rybak, "Stem cells: past, present, and future," Stem Cell Research \& Therapy, vol. 10, no. 1, p. 68, 2019.

[6] P. Taupin, "Stem cells engineering for cell-based therapy," Journal of Neural Engineering, vol. 4, no. 3, pp. R59-R63, 2007.

[7] C. Brown, C. McKee, S. Bakshi et al., "Mesenchymal stem cells: cell therapy and regeneration potential," Journal of Tissue Engineering and Regenerative Medicine, vol. 13, no. 9, pp. 1738-1755, 2019.

[8] H. M. Shahjalal, N. Shiraki, D. Sakano et al., "Generation of insulin-producing $\beta$-like cells from human iPS cells in a defined and completely xeno-free culture system," Journal of Molecular Cell Biology, vol. 6, no. 5, pp. 394-408, 2014.

[9] K. Tateishi, J. He, O. Taranova, G. Liang, A. C. D'Alessio, and Y. Zhang, "Generation of insulin-secreting islet-like clusters from human skin fibroblasts," The Journal of Biological Chemistry, vol. 283, no. 46, pp. 31601-31607, 2008.

[10] D. Van Hoof, K. A. D'Amour, and M. S. German, "Derivation of insulin-producing cells from human embryonic stem cells," Stem Cell Research, vol. 3, no. 2-3, pp. 73-87, 2009.

[11] B. Bose, S. Shenoy P, S. Konda, and P. Wangikar, "Human embryonic stem cell differentiation into insulin secreting $\beta$ cells for diabetes," Cell Biology International, vol. 36, no. 11, pp. 1013-1020, 2012.

[12] M. M. Gabr, M. M. Zakaria, A. F. Refaie et al., "From human mesenchymal stem cells to insulin-producing cells: comparison between bone marrow- and adipose tissue-derived cells," BioMed Research International, vol. 2017, Article ID 3854232, 9 pages, 2017.

[13] G. Daryabor, E. H. Shiri, and E. Kamali-Sarvestani, “A simple method for the generation of insulin producing cells from bone marrow mesenchymal stem cells," In Vitro Cellular \& Developmental Biology, vol. 55, no. 6, pp. 462-471, 2019.

[14] L. Khorsandi, A. Khodadadi, F. Nejad-Dehbashi, and S. Saremy, "Three-dimensional differentiation of adiposederived mesenchymal stem cells into insulin-producing cells," Cell and Tissue Research, vol. 361, no. 3, pp. 745-753, 2015.

[15] T. Teshima, A. Matsuoka, M. Shiba et al., "Comparison of properties of stem cells isolated from adipose tissue and lipomas in dogs," Stem Cells International, vol. 2019, Article ID 1609876, 15 pages, 2019.

[16] T. Teshima, H. Matsumoto, M. Michishita et al., “Allogenic adipose tissue-derived mesenchymal stem cells ameliorate acute hepatic injury in dogs," Stem Cells International, vol. 2017, Article ID 3892514, 12 pages, 2017.

[17] M. M. Gabr, M. M. Zakaria, A. F. Refaie et al., "Generation of insulin-producing cells from human bone marrow-derived mesenchymal stem cells: comparison of three differentiation protocols," BioMed Research International, vol. 2014, Article ID 832736, 9 pages, 2014.

[18] A. Shiroi, M. Yoshikawa, H. Yokota et al., "Identification of insulin-producing cells derived from embryonic stem cells by zinc-chelating dithizone," Stem Cells, vol. 20, no. 4, pp. 284292, 2002. 
[19] T. Ikemoto, R. Feng, M. Shimada et al., "A new 2-step acceleration protocol using a histone deacetylase inhibitor to generate insulin-producing cells from adipose-derived mesenchymal stem cells," Pancreas, vol. 47, no. 4, pp. 477-481, 2018.

[20] S. George, M. R. Hamblin, and H. Abrahamse, "Differentiation of mesenchymal stem cells to neuroglia: in the context of cell signalling," Stem Cell Reviews and Reports, vol. 15, no. 6, pp. 814-826, 2019.

[21] A. Andrzejewska, B. Lukomska, and M. Janowski, "Concise review: mesenchymal stem cells: from roots to boost," Stem Cells, vol. 37, no. 7, pp. 855-864, 2019.

[22] F. al Battah, J. de Kock, T. Vanhaecke, and V. Rogiers, "Current status of human adipose-derived stem cells: differentiation into hepatocyte-like cells," The Scientific World Journal, vol. 11, no. 11, pp. 1568-1581, 2011.

[23] G. Päth, N. Perakakis, C. S. Mantzoros, and J. Seufert, "Stem cells in the treatment of diabetes mellitus - focus on mesenchymal stem cells," Metabolism, vol. 90, pp. 1-15, 2019.

[24] B. M. Abdallah and M. Kassem, "The use of mesenchymal (skeletal) stem cells for treatment of degenerative diseases: current status and future perspectives," Journal of Cellular Physiology, vol. 218, no. 1, pp. 9-12, 2009.

[25] P. Chhabra and K. L. Brayman, "Stem cell therapy to cure type 1 diabetes: from hype to hope," Stem Cells Translational Medicine, vol. 2, no. 5, pp. 328-336, 2013.

[26] M. Liu and Z. C. Han, "Mesenchymal stem cells: biology and clinical potential in type 1 diabetes therapy," Journal of Cellular and Molecular Medicine, vol. 12, no. 4, pp. 1155-1168, 2008.

[27] Y. Zhu, Q. Liu, Z. Zhou, and Y. Ikeda, "PDX1, neurogenin-3, and MAFA: critical transcription regulators for beta cell development and regeneration," Stem Cell Research \& Therapy, vol. 8, no. 1, p. 240, 2017.

[28] R. S. Herbst, "Review of epidermal growth factor receptor biology," International Journal of Radiation Oncology • Biology • Physics, vol. 59, pp. 21-26, 2004.

[29] Y. Sun, L. Chen, X. G. Hou et al., "Differentiation of bone marrow-derived mesenchymal stem cells from diabetic patients into insulin-producing cells in vitro," Chinese Medical Journal, vol. 120, no. 9, pp. 771-776, 2007.

[30] N. Lumelsky, O. Blondel, P. Laeng, I. Velasco, R. Ravin, and R. McKay, "Differentiation of embryonic stem cells to insulin-secreting structures similar to pancreatic islets," Science, vol. 292, no. 5520, pp. 1389-1394, 2001.

[31] D. H. Kassem, M. M. Kamal, A. E. L. G. el-Kholy, and H. O. elMesallamy, "Exendin-4 enhances the differentiation of Wharton's jelly mesenchymal stem cells into insulin-producing cells through activation of various $\beta$-cell markers," Stem Cell Research \& Therapy, vol. 7, no. 1, p. 108, 2016.

[32] T. Brun, I. Franklin, L. St-Onge et al., "The diabetes-linked transcription factor PAX4 promotes $\beta$-cell proliferation and survival in rat and human islets," Journal of Cell Biology, vol. 167, no. 6, pp. 1123-1135, 2004.

[33] J. Liu, Y. Liu, H. Wang et al., "Direct differentiation of hepatic stem-like WB cells into insulin-producing cells using small molecules," Scientific Reports, vol. 3, no. 1, article e1185, 2013.

[34] S. F. Yang, W. J. Xue, Y. F. Duan et al., "Nicotinamide facilitates mesenchymal stem cell differentiation into insulinproducing cells and homing to pancreas in diabetic mice," Transplantation Proceedings, vol. 47, no. 6, pp. 2041-2049, 2015.
[35] R. Wang, N. Yashpal, F. Bacchus, and J. Li, "Hepatocyte growth factor regulates proliferation and differentiation of epithelial monolayers derived from islets of postnatal rat pancreas," Journal of Endocrinology, vol. 183, no. 1, pp. 163-171, 2004.

[36] H. Mashima, H. Shibata, T. Mine, and I. Kojima, "Formation of insulin-producing cells from pancreatic acinar AR42J cells by hepatocyte growth factor," Endocrinology, vol. 137, no. 9, pp. 3969-3976, 1996.

[37] M. B. Sharma, L. S. Limaye, and V. P. Kale, "Mimicking the functional hematopoietic stem cell niche in vitro: recapitulation of marrow physiology by hydrogel-based threedimensional cultures of mesenchymal stromal cells," Haematologica, vol. 97, no. 5, pp. 651-660, 2012.

[38] X. Xin, M. Hussain, and J. J. Mao, "Continuing differentiation of human mesenchymal stem cells and induced chondrogenic and osteogenic lineages in electrospun PLGA nanofiber scaffold," Biomaterials, vol. 28, no. 2, pp. 316-325, 2007.

[39] J. M. Dang, D. D. N. Sun, Y. Shin-Ya, A. N. Sieber, J. P. Kostuik, and K. W. Leong, "Temperature-responsive hydroxybutyl chitosan for the culture of mesenchymal stem cells and intervertebral disk cells," Biomaterials, vol. 27, no. 3, pp. 406-418, 2006.

[40] M. Ravi, V. Paramesh, S. R. Kaviya, E. Anuradha, and F. D. P. Solomon, "3D cell culture systems: advantages and applications," Journal of Cellular Physiology, vol. 230, no. 1, pp. 1626, 2015.

[41] K. Nakamura, R. Iwazawa, and Y. Yoshioka, "Introduction to a new cell transplantation platform via recombinant peptide petaloid pieces and its application to islet transplantation with mesenchymal stem cells," Transplant International, vol. 29, no. 9, pp. 1039-1050, 2016.

[42] K. Nakamura, "CellSaic, a cell aggregate-like technology using recombinant peptide pieces for MSC transplantation," Current Stem Cell Research \& Therapy, vol. 14, no. 1, pp. 52-56, 2019.

[43] A. M. Domouky, A. S. Hegab, A. al-Shahat, and N. Raafat, "Mesenchymal stem cells and differentiated insulin producing cells are new horizons for pancreatic regeneration in type I diabetes mellitus," The International Journal of Biochemistry \& Cell Biology, vol. 87, pp. 77-85, 2017.

[44] T. Ikemoto, R. Feng, S. I. Iwahashi et al., "In vitro and in vivo effects of insulin-producing cells generated by xeno-antigen free 3D culture with RCP piece," Scientific Reports, vol. 9, no. 1, article e10759, Article ID 10759, 2019.

[45] Y. Wada, T. Ikemoto, Y. Morine et al., "The differences in the characteristics of insulin-producing cells using human adipose-tissue derived mesenchymal stem cells from subcutaneous and visceral tissues," Scientific Reports, vol. 9, no. 1, article e13204, Article ID 13204, 2019.

[46] E. D. Rosen and B. M. Spiegelman, "Adipocytes as regulators of energy balance and glucose homeostasis," Nature, vol. 444, no. 7121, pp. 847-853, 2006.

[47] T. Tayaramma, B. Ma, M. Rohde, and H. Mayer, "Chromatinremodeling factors allow differentiation of bone marrow cells into insulin-producing cells," Stem Cells, vol. 24, no. 12, pp. 2858-2867, 2006.

[48] C. Haumaitre, O. Lenoir, and R. Scharfmann, "Histone deacetylase inhibitors modify pancreatic cell fate determination and amplify endocrine progenitors," Molecular and Cellular Biology, vol. 28, no. 20, pp. 6373-6383, 2008. 